\title{
Takotsubo cardiomyopathy after vaccination for coronavirus disease 2019 in a patient on maintenance hemodialysis
}

\author{
Reiko Toida $^{1}$ (1) Shigehiro Uezono ${ }^{2} \cdot$ Hiroyuki Komatsu $^{3} \cdot$ Tatsunori Toida $^{4} \cdot$ Akiko Imamura $^{2} \cdot$ Shouichi Fujimoto $^{4}$. \\ Koichi Kaikita ${ }^{5}$
}

Received: 10 August 2021 / Accepted: 22 October 2021 / Published online: 3 November 2021

(c) Japanese Society of Nephrology 2021

\begin{abstract}
Coronavirus disease-2019 (COVID-19) has affected more than 220 million individuals since the global pandemic began. There is an urgent need for safe and effective vaccines, and vaccinations, such as mRNA vaccines, have been initiated worldwide. However, the adverse effects of these vaccines remain unclear. We herein present a case of an 80-year-old female on maintenance hemodialysis who developed takotsubo cardiomyopathy 4 days after receiving the first dose of the Pfizer-BioNTech COVID-19 vaccine. There was no obvious trigger for the onset of takotsubo cardiomyopathy other than the COVID-19 vaccination, which was the most significant event preceding her presentation. Echocardiograms obtained during her admission allowed us to monitor and show the recovery of left ventricular wall motion. We confirmed the diagnosis of takotsubo cardiomyopathy based on the findings, including transient left ventricular dysfunction, electrocardiographic abnormalities, an elevated troponin level, and the absence of occlusive coronary artery disease. In the present case, the vaccination may have triggered emotional or physical stress. Although difficulties are associated with proving the causal relationship in the present case, the temporal relationship between the vaccination and the onset of takotsubo cardiomyopathy is highly suggestive. The adverse effects associated with the vaccine are typical of COVID-19 vaccines administered to date, most of which are acceptable. Therefore, despite our experience of the present case, we still recommend the vaccination for COVID-19 because takotsubo cardiomyopathy induced by the COVID-19 vaccine is extremely rare and the prognosis of the patient was good. We herein present the first case of a patient on hemodialysis who developed takotsubo cardiomyopathy after receiving COVID-19 vaccination.
\end{abstract}

Keywords COVID-19 vaccine $\cdot$ Adverse effects $\cdot$ Hemodialysis $\cdot$ Takotsubo cardiomyopathy $\cdot$ Left ventricular outflow tract obstruction

Reiko Toida

reiko_toida@med.miyazaki-u.ac.jp

1 Department of Cardiology, Chiyoda Hospital, 88 Furuta, Hichiya, Hyuga city, Miyazaki 883-0064, Japan

2 Department of Nephrology, Chiyoda Hospital, 88 Furuta, Hichiya, Hyuga city, Miyazaki, Japan

3 Faculty of Medicine, Center for Medical Education and Career Development, University of Miyazaki, 5200 Kihara, Kiyotake, Miyazaki city, Miyazaki, Japan

4 Department of Hemovascular Medicine and Artificial Organs, Faculty of Medicine, University of Miyazaki, 5200 Kihara, Kiyotake, Miyazaki city, Miyazaki, Japan

5 Division of Cardiovascular Medicine and Nephrology, Department of Internal Medicine, Faculty of Medicine, University of Miyazaki, 5200 Kihara, Kiyotake, Miyazaki city, Miyazaki, Japan

\section{Introduction}

Coronavirus disease-2019 (COVID-19) has affected more than 220 million individuals and 4.6 million deaths by September 2021. Hemodialysis patients have a mortality rate of more than 20\%, and mortality from COVID-19 has been twofold higher in the hemodialysis population (median age 71.7 years) than in age-matched nonhemodialysis patients with COVID-19 [1]. There is an urgent need for safe and effective vaccines, and vaccinations, such as mRNA vaccines, have been initiated worldwide. However, the adverse effects of these vaccines remain unclear.

We herein report the first case of a patient on maintenance hemodialysis who developed takotsubo cardiomyopathy with left ventricular outflow tract obstruction after receiving the COVID-19 vaccination. 


\section{Case report}

An 80-year-old woman was admitted with hypotension during dialysis. The patient had been receiving hemodialysis for two and half years due to renal sclerosis. She had been prescribed amlodipine for hypertension and calcitriol and calcium carbonate for secondary hyperparathyroidism with hyperphosphatemia. Her husband died last year and she lived alone. The patient had not experienced any recent emotional or physical stress and did not take supplements or drink alcohol. She did not have any known allergies to food, drugs, or other vaccines. Furthermore, she did not have a family history of heart disease. Four days before admission, the patient had received the first dose of the Pfizer-BioNTech COVID-19 vaccine. The day after the vaccination, she developed general fatigue and loss of appetite. At admission, systolic/diastolic blood pressure was $82 / 47 \mathrm{mmHg}$, and heart rate was $114 \mathrm{bpm}$ with an irregular rhythm. A physical examination revealed a systolic murmur of Levine $2 / 6$ at the second left sternal border, clear lung sounds, and no leg edema. On admission, electrocardiography (ECG) newly revealed atrial fibrillation with a normal axis, negative T-waves in I, aVL, and V3-6, and a prolonged QTc interval of $495 \mathrm{~ms}$ (Fig. 1). There were no abnormal findings on ECG other than a high voltage in the left ventricle (LV) on routine ECG before the onset of the disease. Laboratory tests revealed a significantly elevated troponin-I level of $2264 \mathrm{pg} / \mathrm{mL}$ (nor$\mathrm{mal} \leq 30 \mathrm{pg} / \mathrm{mL}$ ). Other blood tests, including a complete blood count, the eosinophilic-leukocyte ratio, creatine kinase-muscle/brain, blood sugar levels, and C-reactive protein levels, were within normal limits.
Transthoracic echocardiography performed approximately 11 months before the onset of the disease did not show any findings suggestive of LV enlargement, diastolic dysfunction, or systolic dysfunction. In admission, transthoracic echocardiography revealed akinesia of the apical segments of the LV with apical ballooning and sparing of the base of each wall as well as a reduced ejection fraction of $48 \%$. Systolic anterior motion of the mitral valve (MV) and LV outflow tract obstruction with basal hyperkinesia were detected in addition to mild-moderate mitral regurgitation. Peak flow velocity and the mean pressure gradient of the LV outflow tract were $4.2 \mathrm{~m} / \mathrm{s}$ and $71 \mathrm{mmHg}$, respectively (Fig. 2). These findings were newly detected. The annular calcification of MV was heavy and accumulated as a mass in the posterior lobe, and these findings did not markedly differ from those obtained in a previous examination in June 2020. Coronary computed tomography showed no significant stenosis of the coronary arteries and extensive akinesis in the apical portion and hyperkinesia in the basal portion of $\mathrm{LV}$ with apical ballooning (Fig. 3).

We confirmed the diagnosis of takotsubo cardiomyopathy based on the above findings and treated the patient with oxygen and the intravenous administration of a small amount of fluid. Although there was a risk of LV thrombosis, anticoagulant therapy was not performed because the patient was receiving dialysis [2].

The day after admission, symptoms improved and ECG showed a sinus rhythm, but ST changes remained unchanged. On day 5, ECG showed deeper negative T-waves and a prolonged QTc interval of $510 \mathrm{~ms}$. The level of troponin-I decreased from that on admission $(352 \mathrm{pg} / \mathrm{mL})$. Echocardiography showed normalized contractility of the

Fig. 1 Initial electrocardiogram

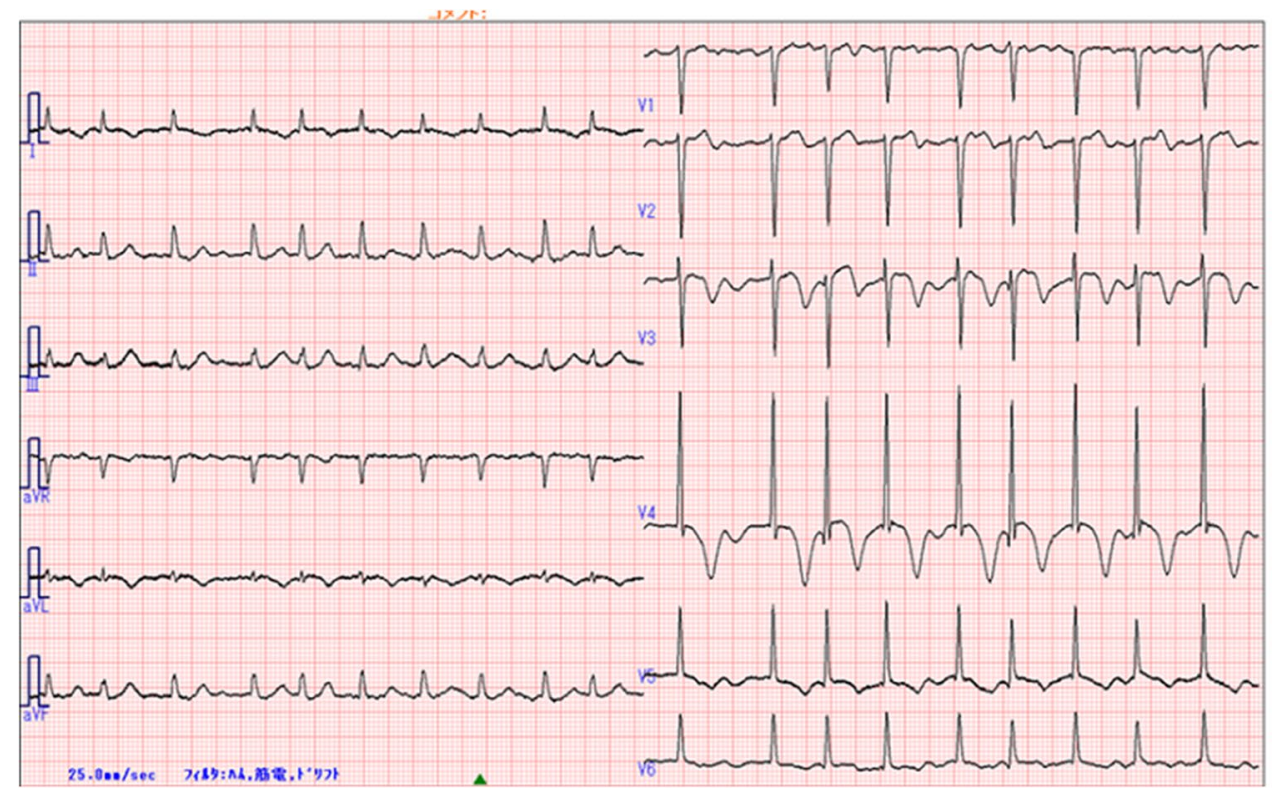




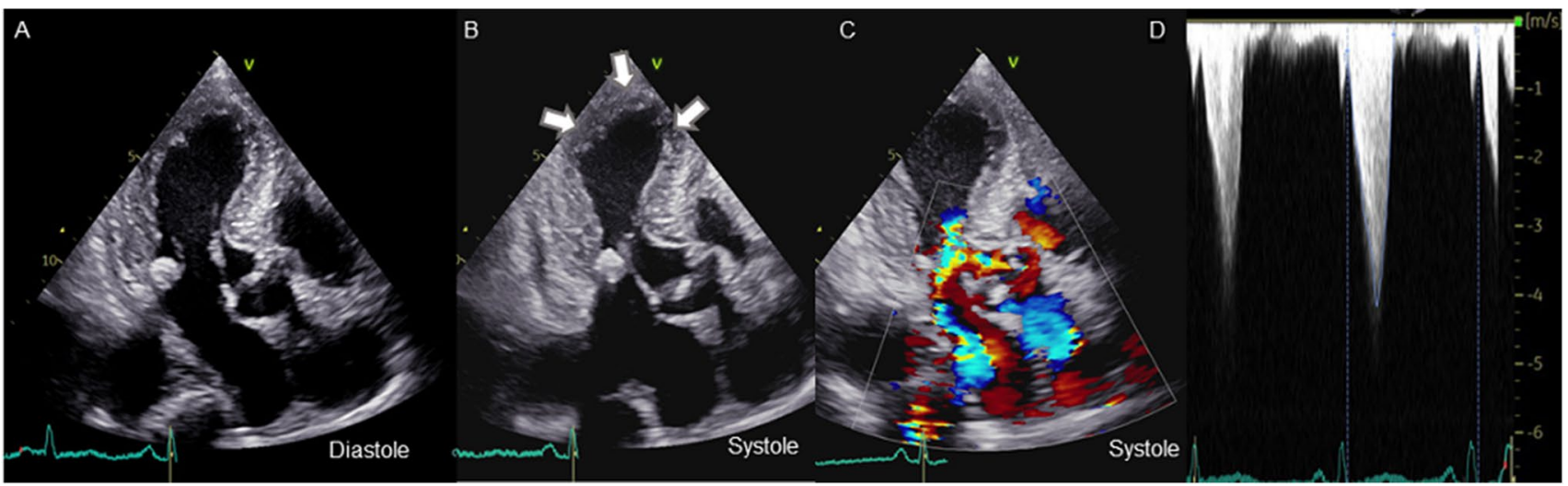

Fig. 2 Echocardiography of akinesia of apical segments of the left ventricle (LV) and LV outflow tract systolic anterior motion of the mitral valve (MV) with basal hyperkinesia was observed with mildmoderate mitral regurgitation. Apical three-chamber view at diastole

(A) and systole (B) using echocardiography. Color Doppler image of the LV outflow tract at systole $(\mathbf{C})$ and the flow velocity waveform (D)

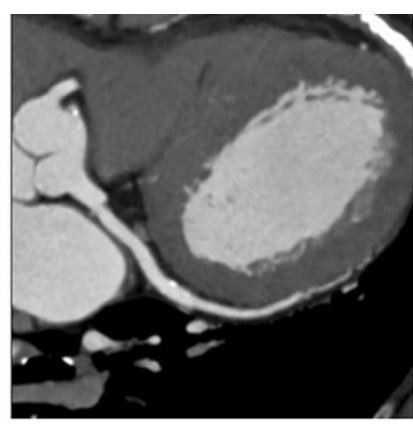

LCX

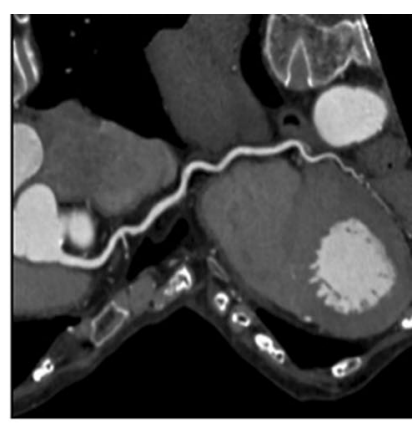

RCA apical myocardial segment, with the normalization of LV ejection fraction systolic function of $63 \%$, as expected with takotsubo cardiomyopathy. LV outflow tract obstruction were not detected. Systolic blood pressure recovered to $120 \mathrm{mmHg}$ and the patient was discharged on day 13 without any complications. Although it was not possible to rule out coronary artery disease based on the symptoms, the findings of a physical examination, or ECG and echocardiography findings, it was not detected on coronary computed tomography. Furthermore, improvements in cardiac function after a few days supported the diagnosis of takotsubo cardiomyopathy. One month after the diagnosis of takotsubo cardiomyopathy, the ST changes on ECG had improved and LV wall motion and ejection fraction by echocardiography remained normalized. The patient is receiving maintenance hemodialysis without hypotension or arrhythmia.

\section{Discussion}

We herein presented the first case of a patient on hemodialysis who developed takotsubo cardiomyopathy with LV outflow tract obstruction after receiving the COVID-19 vaccination.

Since the initial outbreak in December 2019, COVID19 has spread rapidly across the world, causing a global pandemic. COVID-19 has affected more than 220 million people and killed 4.6 million by September 2021. It is also now well recognized that COVID-19 has a significant impact on people undergoing maintenance dialysis. The overall case fatality rate is $20-30 \%$, coupled with the risk of exposure, especially in people undergoing hemodialysis $[1,4]$. Therefore, there is an urgent need for safe and effective vaccines and immunizations, especially for compromised hosts, such as hemodialysis patients. 
The findings of a phase III study on the mRNA-1273 SARS-CoV-2 vaccine suggested high efficacy with an excellent safety profile [3]. Blake et al. reported that SARS-CoV-2 infection was less frequent and less severe in vaccinated dialysis patients and also that two doses were more effective, both for the frequency and intensity of the serologic response, than one dose [4]. The majority of adverse effects, including injection site reactions, malaise, fatigue, and headache, have been mild and attributed to the response of the immune system [3].

A large cohort study performed at the Cleveland Clinic showed that the incidence of takotsubo cardiomyopathy has increased within the general population during the COVID19 pandemic. These findings indicate that the psychological, social, and economic stresses accompanying the pandemic are more likely to be contributing to the increased number of takotsubo cardiomyopathy cases than direct viral involvement and the sequelae of infection [5]. Another study reported an increased incidence of takotsubo cardiomyopathy in COVID-19 patients, which may be associated with the cytokine storm, increased sympathetic responses, and microvascular dysfunction [6].

Takotsubo cardiomyopathy is a clinical syndrome characterized by an acute and transient ( $<21$ days) LV systolic (and diastolic) dysfunction often related to an emotional or physical stressful event, most often identified in the preceding days (1-5 days). It is more likely to occur in postmenopausal women and its pathophysiology is considered to be associated with elevated catecholamine levels, resulting in microvascular spasms and myocardial dysfunction [2]. Well-accepted takotsubo cardiomyopathy diagnostic criteria are from the Mayo Clinic and consist of four components: (1) temporary hypokinesis, dyskinesis, or akinesis in LV segments with or without apical involvement; aberrations in regional wall motion exceeding a single vascular distribution; the existence of stress elicitation; (2) the lack of significant coronary artery disease; (3) recent changes on ECG (ST-segment elevation and/or T-wave inversion) or a significant elevation in serum cardiac troponins; and (4) the absence of pheochromocytoma or myocarditis [2]. Based on these diagnostic criteria, we considered the present case to be compatible with a diagnosis of takotsubo cardiomyopathy.

In the present case, there was no obvious trigger for the onset of takotsubo cardiomyopathy other than the COVID19 vaccination, which was the most significant event preceding her presentation. The temporal relationship between the vaccination and the onset of takotsubo cardiomyopathy is highly suggestive. The underlying mechanisms for the onset of takotsubo cardiomyopathy after the vaccination remain unclear. Jani et al. described a previous case of takotsubo cardiomyopathy after the influenza vaccination and speculated that a similar mechanism related to a sympathovagal imbalance secondary to the vaccine may lead to the development of takotsubo cardiomyopathy after the COVID-19 vaccination [7]. This hypothesis may be applicable to the present case.

We strongly support the efficiency of the COVID-19 vaccination to control the pandemic because takotsubo cardiomyopathy induced by COVID-19 vaccine is extremely rare, the adverse effects associated with the Pfizer-BioNTech COVID-19 vaccine are typical of COVID-19 vaccines administered to date, most of which are acceptable [8] and COVID-19 itself may induce serious cardiovascular sequelae, such as severe thrombosis.

Takotsubo cardiomyopathy in this report may be one of the complications that might be considered in people who have chest symptoms and hypotension after COVID-19 vaccination. In the future, these rare adverse effects will provide a more detailed understanding of responses to the COVID19 vaccination and similar mRNA vaccines. The careful follow-up of patients after vaccinations is still important.

Acknowledgements We acknowledge Momoko Konami, Saki Kashiwada, and Hiroko Tanaka (clinical laboratory technicians in Chiyoda Hospital) for their assistance in clinical data collection for this article.

\section{Declarations}

Conflict of interest The authors have declared that no conflict of interest exists.

Ethical approval All procedures performed in studies involving human participants were in accordance with the ethical standards of the institutional and/or national research committee at which the studies were conducted and with the 1964 Helsinki declaration and its later amendments or comparable ethical standards.

Informed consent The patient provided informed consent for the publication of this case report.

\section{References}

1. Jager KJ, Kramer A, Chesnaye NC, Couchoud C, Sánchez-Álvarez JE, Garneata L, et al. Results from the ERA-EDTA registry indicate a high mortality due to COVID-19 in dialysis patients and kidney transplant recipients across Europe. Kidney Int. 2020;98:1540-8.

2. Medina de Chazal H, Del Buono MG, Keyser-Marcus L, Ma L, Moeller FG, Berrocal D, et al. Stress cardiomyopathy diagnosis and treatment: JACC state-of-the-art review. J Am Coll Cardiol. 2018;72:1955-71.

3. Baden LR, El Sahly HM, Essink B, Kotloff K, Frey S, Novak R, et al. Efficacy and safety of the mRNA-1273 SARS-CoV-2 vaccine. N Engl J Med. 2021;384:403-16.

4. PG Blake, MA Hladunewich, MJ Oliver. COVID-19 Vaccination Imperatives in People on Maintenance Dialysis. Clin J Am Soc Nephrol. 2021; CJN.07260521 
5. Jabri A, Kalra A, Kumar A, Alameh A, Adroja S, Bashir H, et al. Incidence of stress cardiomyopathy during the coronavirus disease 2019 pandemic. JAMA Netw Open. 2020;3:e2014780.

6. Shah RM, Shah M, Shah S, Li A, Jauhar S. Takotsubo syndrome and COVID-19: associations and implications. Curr Probl Cardiol. 2021;46:100763.

7. Jani C, Leavitt J, Al Omari CO, Pond K, Gannon S, Chandran Kumar A, et al. COVID-19 vaccine-associated takotsubo cardiomyopathy. Am J Ther. 2021;28(3):361-4.
8. CDC COVID-19 Response Team, Food and Drug Administration. Allergic reactions including anaphylaxis after receipt of the first dose of Pfizer-BioNTech COVID-19 vaccine-United States, December 14-23, 2020. Morb Mortal Wkly Rep. 2021;70:46-51.

Publisher's Note Springer Nature remains neutral with regard to jurisdictional claims in published maps and institutional affiliations. 